\title{
Scanning capacitance microscopy of TGS - TGS + Cr ferroelectric crystals
}

R.V. Gainutdinov ${ }^{1}$, N.V. Belugina ${ }^{1}$, A.K. Lashkova ${ }^{1}$, K.L. Sorokina ${ }^{1}$, V.N. Shut ${ }^{2}$, I.F. Kashevich ${ }^{3}$, S.E. Mozzharov ${ }^{2}$, A.L. Tolstikhina ${ }^{1}$

${ }^{1}$ Shubnikov Institute of Crystallography of Federal Scientific Research Centre "Crystallography and Photonics" of Russian Academy of Sciences, 119333, Leninsky pr. 59, Moscow, Russia

e-mail:aklashkova@yandex.ru

${ }^{2}$ Institute of Technical Acoustic NASB, 210027, Vitebsk, Belarus

${ }^{3}$ Vitebsk State University, 210032, Vitebsk, Belarus

Scanning capacitance microscopy (SCM) is known as a method for measuring spatial variations in capacitance with high resolution. It is mainly used for high-precision analysis of the dopant concentration in semiconductor structures, for the quantitative evaluation of the dielectric films thickness or for the dielectric constant [1]. To the ferroelectrics so far this method has been applied little, as one can see from the limited number of research papers [2,3].

The main objects of study were TGS-TGS + Cr crystals with a profile impurity distribution of chromium ions grown at the Institute of Technical Acoustics NASB [4]. The banded crystals TGS-TGS + Cr were studied in the work by SCM, PFM, KPFM methods. A periodic change in the composition was achieved by growing the seed in solutions of a different composition (nominally pure and containing an admixture of $\mathrm{Cr}^{3+}$ (5-6\% by weight)). Difference in concentration of $\mathrm{Cr}^{3+}$ between nominally pure and containing an impurity stripes turns out $\sim 0.08 \%$ by weight.

Combining a large number of consecutive images into one allows to imagine the general situation of the domain structure in different stripes. Figure 1 shows the panoramic image composed of 16 PFM images. The domain structure of nominally pure and containing an impurity stripes is markedly different. Fine-dispersed domain structure corresponds to the impurity areas.

On PFM image, the contrast is formed only on the positive and negative domains (Fig. 2a). The presence of the fine-dispersed domains on one side of the boundary and in the stripe itself indicates an impurity area. The same situation is repetitive in the SCM image (Fig. 2b). This can be evidence of the existence of a capacitive contrast associated with the domain structure. In this case three contrasts are observed: light (corresponding to domain boundaries), dark (corresponding to the stripe of pure TGS) and medium (corresponding to the stripe with impurity). Medium contrast in our case is due to the presence of an impurity.

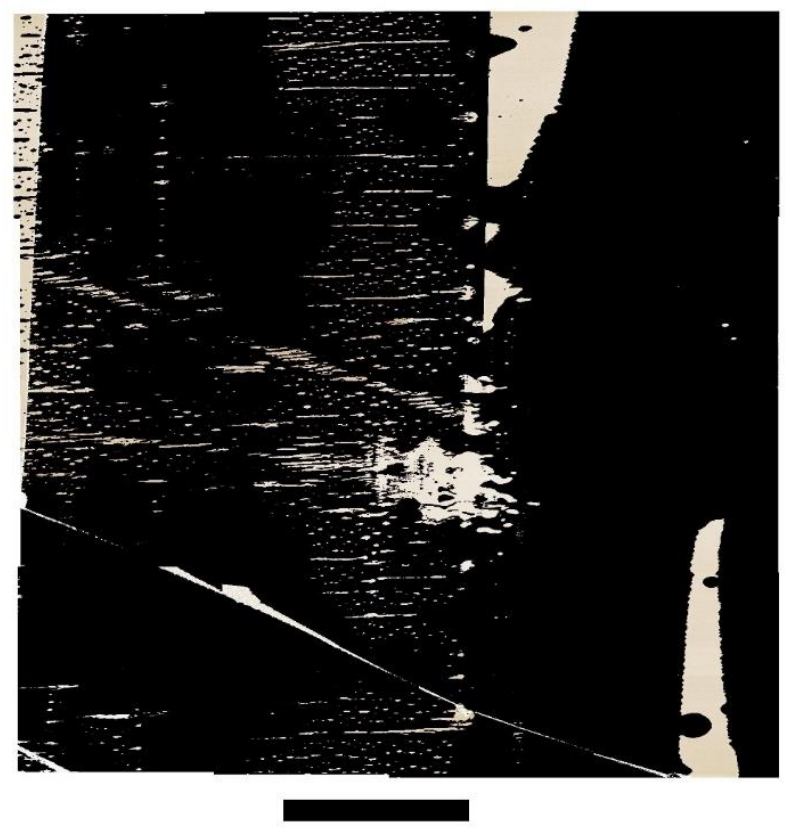

$80 \mu \mathrm{m}$

Figure 1. Panoramic PFM image of the domain structure of TGS-TGS + Cr crystals. 


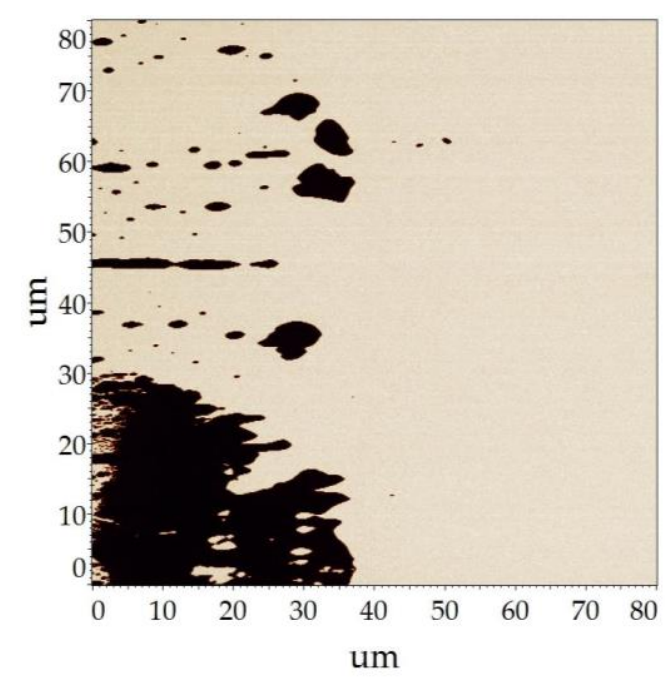

(a)

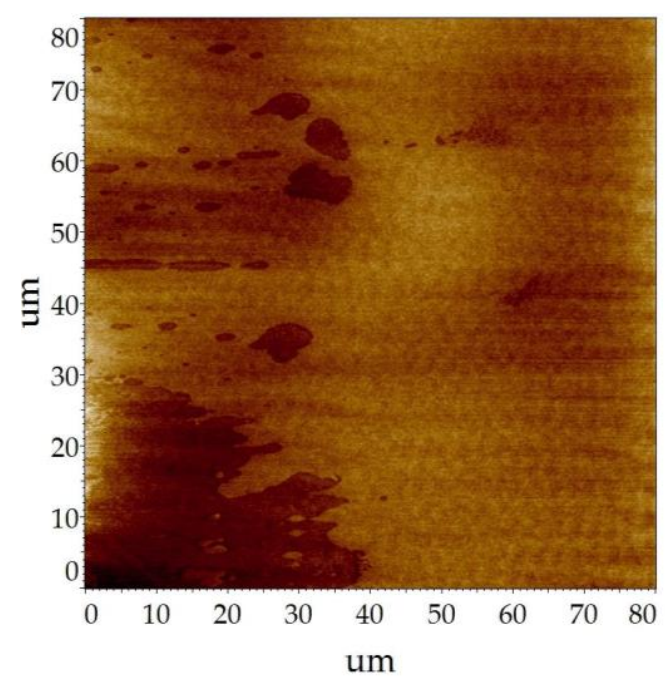

(c)
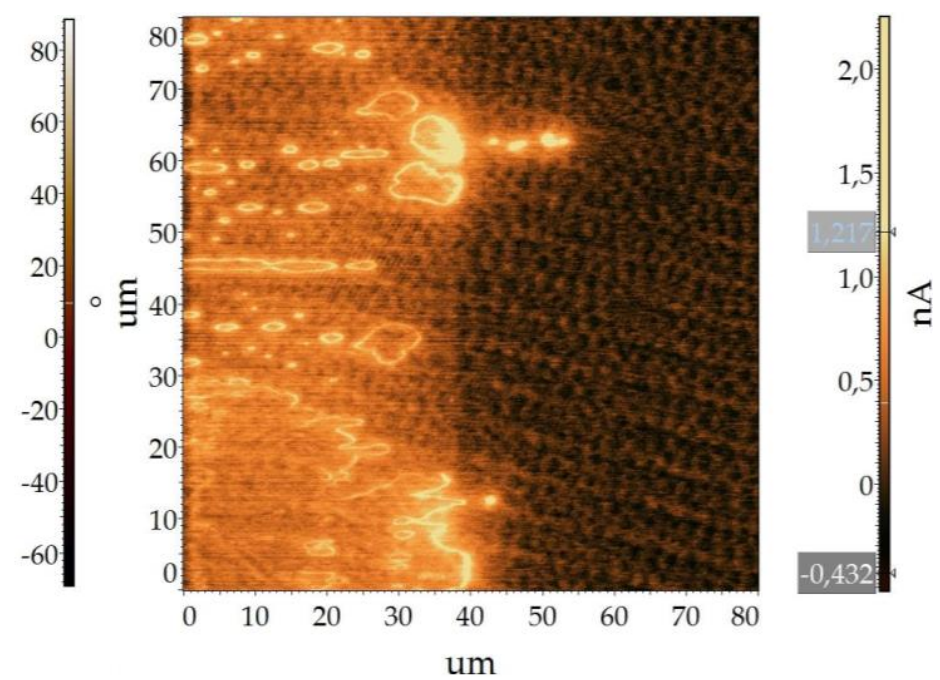

(b)
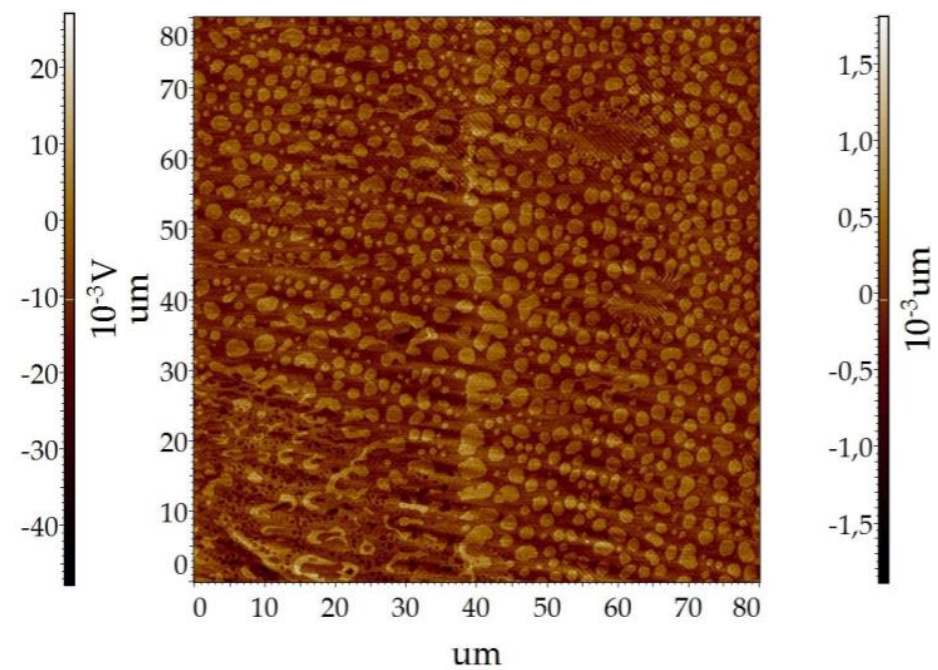

Figure 2. Image of the same surface area of TGS - TGS $+\mathrm{Cr}$ with containing an impurity of Cr3+ stripe (left) and nominally pure TGS (right): (a) PFM, (b) SCM, (c) KPFM, (d) corresponding surface relief. Size $80 \times 80 \mu \mathrm{m}$.

This shows that the SCM method is more informative than PFM and KPFM, because they do not have sensitivity to the presence of impurities in the crystal. If there is no characteristic finedispersed domain structure on the researching surface, these methods will not show the boundary of the impurity stripe.

It is demonstrated that SCM is an effective method for controlling the spatial variation of the local permittivity and studying the relationship between the features of the domain structure and the impurity composition of local regions of ferroelectric crystals with a profile impurity distribution. Measurements with the help of the B2987A petaohmmeter at the Cascade Microtech Tesla probe station do indeed show 1.5-2 times larger current value for the impurity regions of the surface at ohmic regions of volt-ampere characteristics in comparison with the pure ones.

1. B. Bhunshan, H. Fuchs, M. Tomitori, Applied Scanning Probe Methods YIII: Scanning Probe Microscopy Techniques (Springer-Verlag), (2008).

2. C.-C. Leu et al. Electrochem. Solid-State Lett 7, A327 (2004).

3. Y. Naitou, H. Ogiso. Jap. J. Appl. Phys. 45, 1922 (2006).

4. V.N. Shut, I.F. Kashevich, B.E. Votts, Crystallogr.Rep. 49, 206 (2004). 